\title{
Multiple Architectural Approach for Urban Development Using Wearable IoT Devices: A Combined Machine Learning Approach
}

\author{
Raghu T. Mylavarapu', Bharadwaja Krishnadev Mylavarapu² \\ ${ }^{1}$ Regional Development Center, KPMG LLP, Montvale, NJ, USA \\ ${ }^{2}$ Business Analytics Lead, Department of Analytics, PLM COE, PDM, John Deere Co., Moline, IL, USA \\ Email: rmylavarapu@kpmg.com, bharadwajakrishnadev@johndeere.com
}

How to cite this paper: Mylavarapu, R.T. and Mylavarapu, B.K. (2018) Multiple Architectural Approach for Urban Development Using Wearable IoT Devices: A Combined Machine Learning Approach. Advances in Internet of Things, 8, 27-38. https://doi.org/10.4236/ait.2018.83003

Received: March 22, 2018

Accepted: July 28, 2018

Published: July 31, 2018

Copyright ( $) 2018$ by authors and Scientific Research Publishing Inc. This work is licensed under the Creative Commons Attribution International License (CC BY 4.0).

http://creativecommons.org/licenses/by/4.0/

\begin{abstract}
Machine Learning becomes a part of our life in recent days and everything we do in interlinked with machine learning. As a technocrat, we tried to implement machine learning with Internet of Things (IoT) for better implementation of technology in organizations for security. We designed an sample architecture which will carry the burden of safeguarding the organizational data with IoT using machine learning with an effective manner and in this case we were proposing utilization of cloud computing for better understanding of data storage and retrieval process. Machine learning is used for the prediction models based on which we need to perform high level analysis of data and using IoT we promote authorization mechanism based on which we recognize the appropriate recipient of data and cloud for managing the data services with the three-tier architecture. We present the architecture we are proposing for better utilization of machine learning and IoT with cloud architecture.
\end{abstract}

\section{Keywords}

Cloud Computing, Machine Learning, IoT, Authentication, Architecture

\section{Introduction}

Machine learning in real-time scenario plays a crucial role and we need to concentrate on how to improve the technical architecture for the security systems with high level data transmission and protection, how the data is transferred from one device or sensor to the repository and how the data is managed and how to get and update the information. Here we are proposing a four-tier architecture which will create a revolutionary implementation in science and tech- 
nology related to ML and AI. In [1], authors discussed about the concept of implementation of IoT and cloud in urban development which leads to utilization of different mechanisms in machine learning. The goals the authors discussed in this article are regarding identifying the low cost implementation, effective data collection, robust methodology, globally acceptable thing. These are the main focus things of authors mentioned in that article. Wearable devices tackle the information with from sensors of the devices and transmit them to the server or the repository in cloud. For providing services for the wearable devices, we need to have large setup and we need permission from the cellular network provider [2], or we need to gather or create the custom applications which are written in mobile platforms support [3]. We need to consider the security measure that how we can utilize the personal data without causing any security bleach [4]. In this architecture, we have four stages and in the first stage, we have a sensor which will trap the all the data from the surrounding environment, in the second stage, we have a middleware like a data converter which will convert the data captured by the sensor into human readable language which will be having the combination of IoT and ML. In this phase we need to translate the things using ML algorithms like NLP [5]-[10]. Natural Language Processing is a mechanism using which we will convert the unstructured data to semi structured or structured data. Third face will have the cloud repository using which we need to store and manage the data and we don't have much pressure in working on it. Instead of establishing repository we can leverage resources from the third party services like AWS, GCP etc., in the fourth phase we have to gather all the data and process it and find the result we want [11]-[16].

Our intension here is very clear and we are trying to design and implement generic architecture which will fit for IoT and ML devices for better prediction. In the later sections, we discuss about the domain of implementation, what are the challenges is highlighted in that domain, in next section, we discuss about the individual tiers of implementation, later section deals with the architecture explanation, final section is with expected results and conclusion.

\section{Domain of Implementation}

\subsection{Urban Development}

In this urban development we need to consider the defects around you to understand what the people need and there are any security measures we need to consider to explain to the higher officials. In this urban development as the survey conducted by open sources these are the challenges they are facing.

Lake of proper security for women;

Road damage;

$>$ Improper constructions;

$>$ Water problem;

Education system;

Traffic issue; 
Medical problems;

Electricity;

Food;

Human Mafia.

In all these things consider the issues what can be identified by the sensors of the wearable devices. The issues like security, road damage, traffic, electricity and human mafia. Let's discuss everything in details as below.

\subsubsection{Security}

People while traveling or walking on roads they need to wear a device which is connected to mobile with a pre installed app through Bluetooth and mobile has to be connected to the repository through mobile data. Some people may think about the battery draining of the device because it have to be connected to server, but the thing here is this is a mandatory thing in our architecture [17] [18] [19] [20] [21]. Using the wearable device we can take images on the road and they will be transferred to the mobile and those will not be shared in the device and will directly transferred to the central server in the cloud repository of the agency. There based on the requirement we need to process those images and clips. Our intelligent machine has to identify what's happening in that image. This will happen only with machine learning and AI [22] [23] [24].

\subsubsection{Road Damage}

Images from device related to road will be captured and sent to central server, then based on the image, we satellite over view will come to the machine and identify where that road is located and based on that information, specific department will be alerted for recovery of the road and solve it as soon as possible [25].

\subsubsection{Traffic}

Traffic system will be maintained by taking images of current location and update into the central traffic system server. It will update to all the people in the surrounding locality to avoid the traffic disturbances [26] [27].

\subsubsection{Electricity}

Electricity issues are more in the rural areas as well as interiors in the urban areas and people may not able to communicate to the officials about their issue [25] [28] [29] [30]. In this way using the wearable device we are proposing you can transfer the information in the form of chain link from one device to another device $t$ o intimate the issue to the respective department. Regarding the architecture we will discuss in the lateral sections clearly.

In the above detailed analysis we have different challenges in urban area development using IoT wearable devices which will transmit the data. Here we are only proposing the architecture and not prescribing any networking scenario to transfer the data. And this can be considered as the future extension of this current scenario we are following [31]. 
Using this proposed architecture we are overcoming the existing system issues like efficient utilization of technology and we are proposing advanced framework of the data sharing and problem solving mechanism. In the next section we are going to discuss about the current scenarios in market and finally we will explain the proposed architecture.

\section{Existing Architecture}

The following architecture is the present existing architecture and we are using SENSg technology proposed in the existing architecture to transfer the data from wearable devices to the repository. The following image will explain the concept of existing architecture using SENSg [1] as shown in Figure 1.

The present approach consisting of the SENSg technology which was proposed by [1] and this will lead to take utilization of cloud and API's [5] [6] for better implementation of data transfer in a better way. The current scenario will connect the device with internet and using the API's data is transferred to cloud and they are maintained for further implementations. But the thing here is we need to utilize temperature pressure control devices for effective maintenance of the device and architecture [32].

Figure 2 will define the IoT device architecture which is as follows.

Here the existing work authors use two types of PCB's. One is single sided v4 $\mathrm{PCB}$ and other is double sided v3 PCB as shown in Figure 3.

\section{Proposed Architecture}

In this proposed architecture we are focusing on implementing ML with IoT with four tier architecture. In this architecture we are having four different regions and they are explained here below.

\subsection{Wearable IoT Device}

A wearable IoT device like Figure 4 mentioned below will have the inbuilt camera and the controller fixed to it. Using controller, person will take pictures of the issues around him and upload that to the server through tier 2. It's an IoT network Controller.

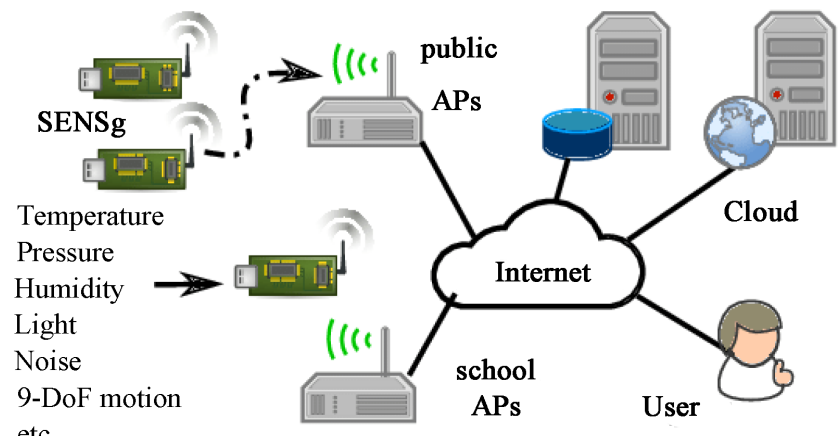

Figure 1. SENSg Architecture. 

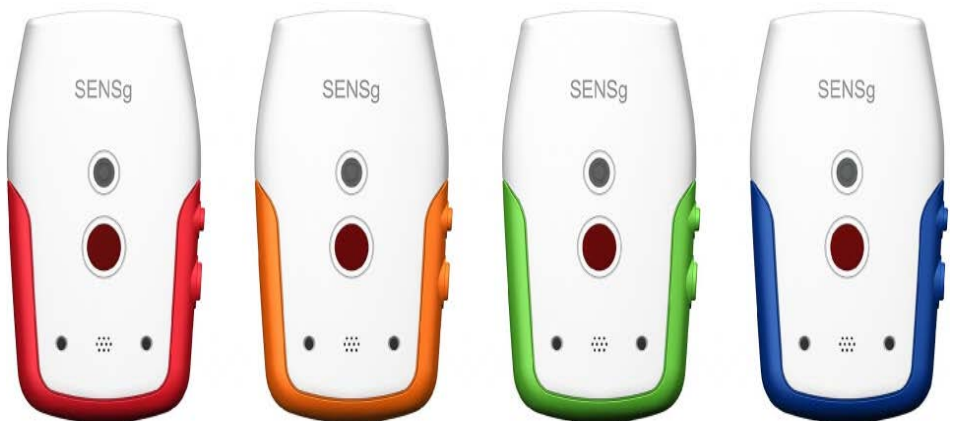

Figure 2. IoT device in use.
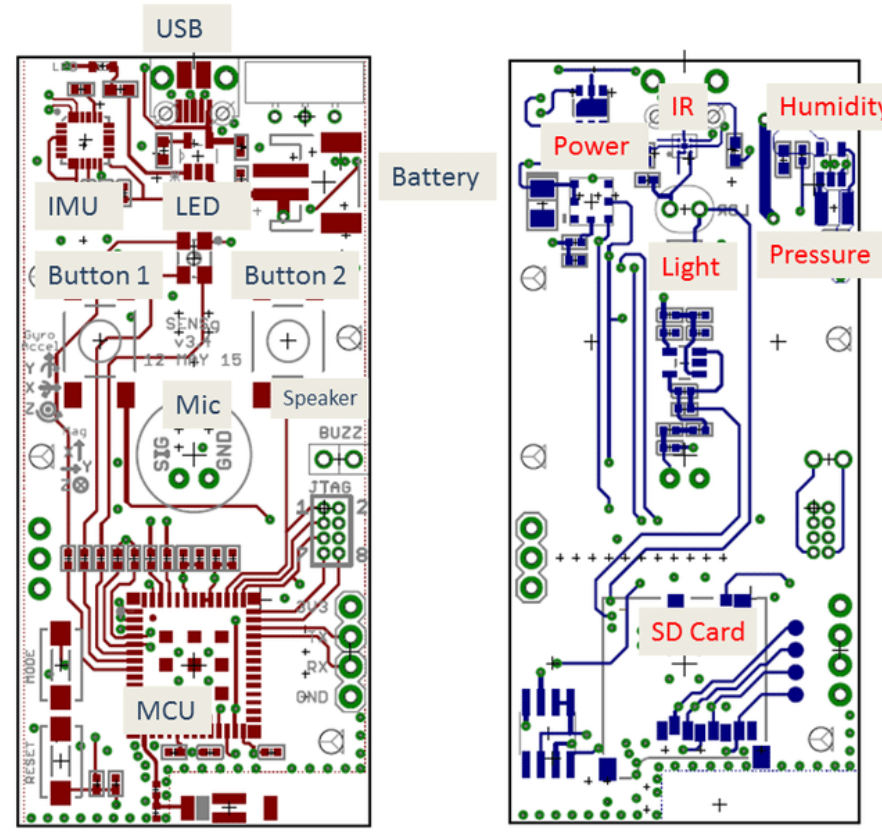

Antenna

Figure 3. Double Sided PCB for V3 board.

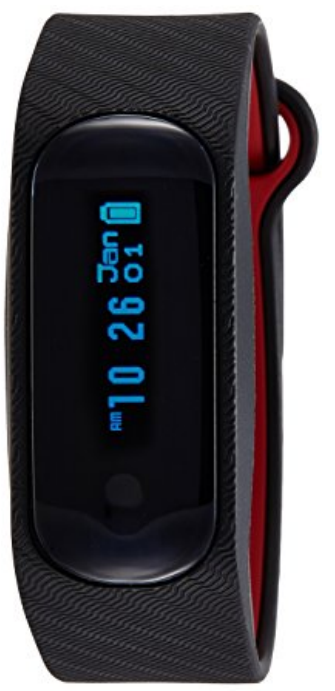

Figure 4. IoT device which have capability of taking images (Proposing Design). 
This wearable device will capture the image by clicking and we can't see the clicked images and they will automatically move to device in which app is being controled. This is tier 2 and it is resembled in this paper as IoT networks [2] [3] [4] [5].

\subsection{IoT Network (Application Layer)}

In this tier we are discussing about the how the data is transferred to the device in which application is installed how we are not storing information in device even not as temporary data. Here using the IoT networks the data transferred from device to the cloud. In this stage we are proving security measures to maintain high security of the information which is in the transmission. The main cause of separating networking pattern is here we are considering application in the mobile device and the reason behind not storing information in device is to maintain security. This information from IoT device has to transfer directly to the server but it has to be through a medium. That's the reason why we are using application in mobile and transmitting information through application [6] [7] [8].

Figure 5 will resemble the network point of the IoT device and this will be considered as the tier 2 of the architecture.

\subsection{Cloud Storage (Database Layer)}

The database layer consists of the information we transferred from the application to the cloud storage in the remote location. We have the sample network in cloud storage and we have to take network access from CSP (Cloud Service Provider) to manage the access.

\subsection{Machine Learning (Predictions Phase)}

Machine Learning is for designing prediction models through various algorithms available. To convert image or video to text we need to use NLP and based on the information gathered we need to transfer the specific issue to the respective department [15].

Here is the sample architecture of NLP in machine learning as shown in Figure 6.

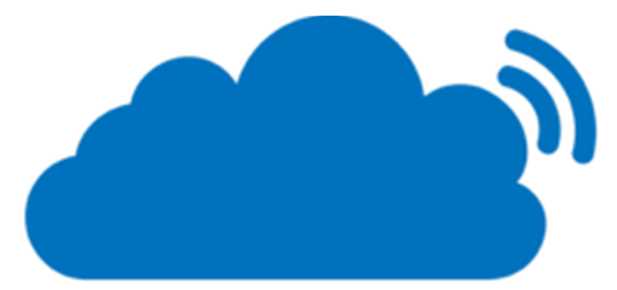

\section{THE THINGS N E T W O R K}

Figure 5. Enchrypted IoT network through application. 


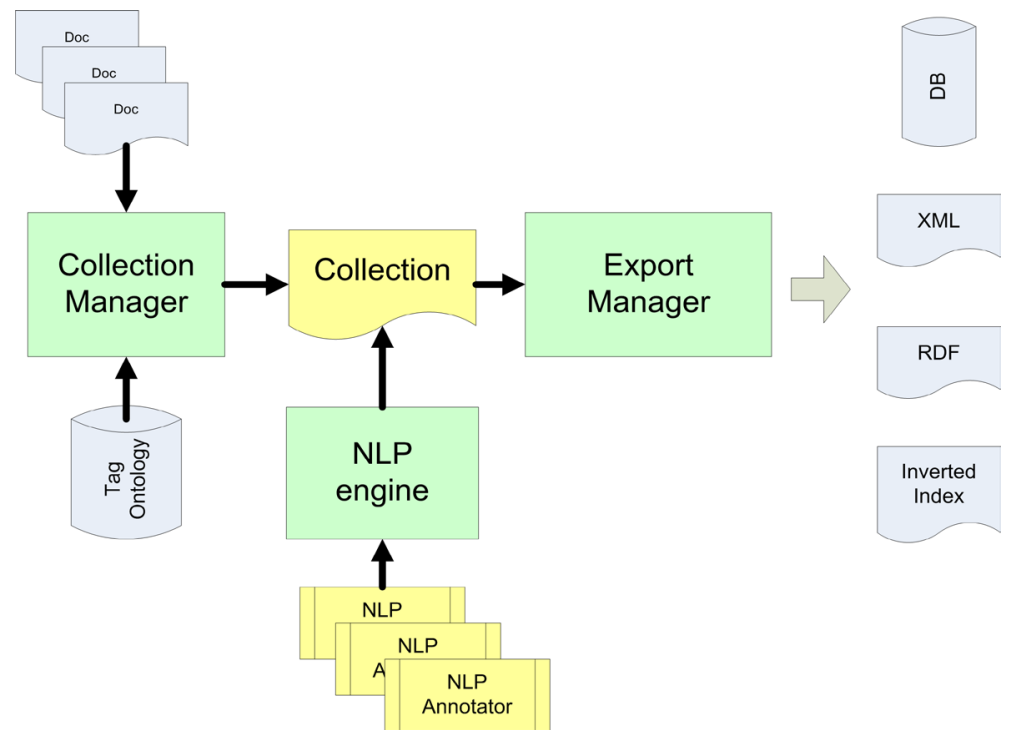

Figure 6. NLP Architecture.

By considering all the above tiers we are prescribing one architecture which will solve the problems in urban development using wearable devices in ML and IoT combination with the support of Cloud Computing as shown in Figure 7.

\section{Implementation and Results}

- We can take high pixel images with this device with the help of camera inbuilt it.

- High secured data transmission and data is transferred through encrypted file and we can do that encryption using RSA algorithm.

- High level predictions will happen with NLP converted data. We can predict the high chances of issues based on locality.

- High accuracy in predictions.

- High utilization of Cloud architecture and services.

\subsection{Implementation Procedure}

Implementing this kind of architecture is typical task and we are succeeded in this and we are up to some valuable extent and the details are as follows.

\subsubsection{IoT Device Architecture}

IoT device architecture will be different from other devices. I will consist of a camera sensor and which can take images with a click on the controller. We have to control that device with Bluetooth connection to the mobile device in which the device application is running in background. Here we use Bluetooth protocol BLE (Bluetooth Low Energy). This protocol is from Bluetooth V4 and this uses low energy from the devices and this will be using for this architecture and this will used by the wearable device and the data is transferred through that protocol. For example consider a hospital management through wearable device. Patient information will be transferred to the server and then to doctors premises. 


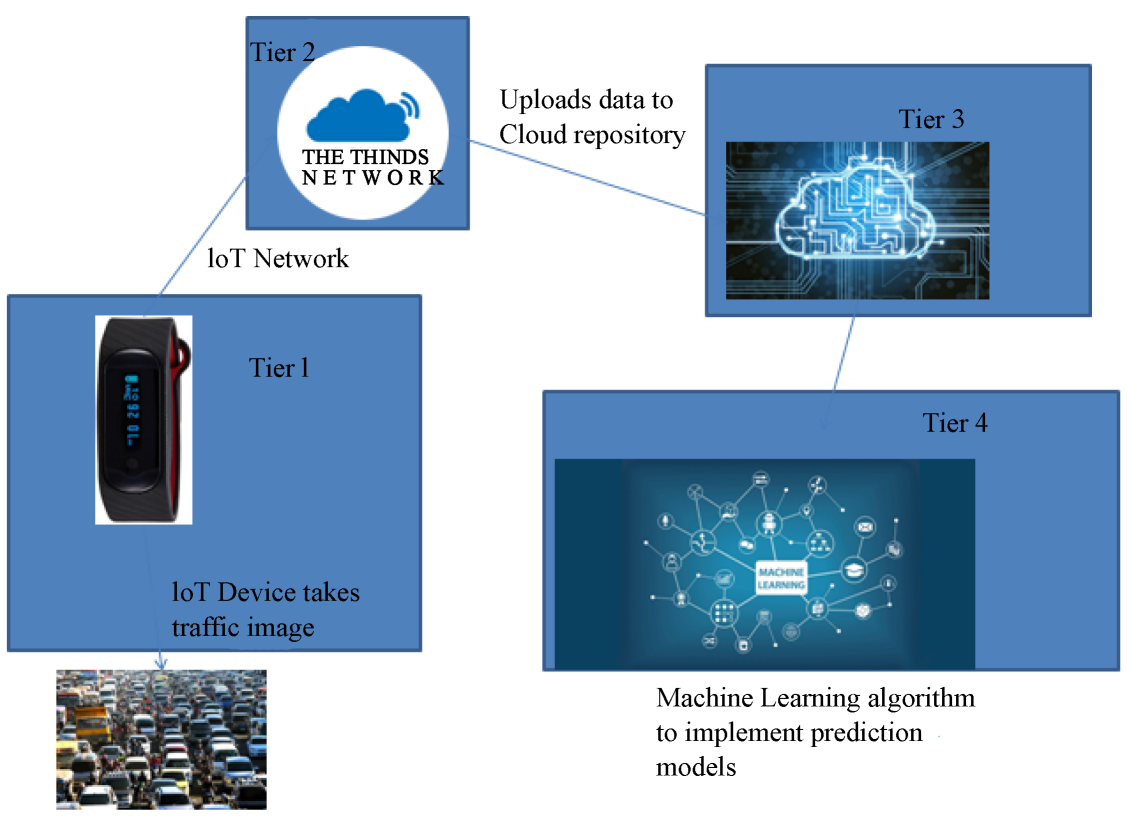

Figure 7. Prescribed urban development architecture.

BLE protocol is based on GATT which means Generic Attribute. This defines how the two devices will connect to the low energy bandwidth. Like standard Bluetooth architecture we need concepts like services and characteristics.

\subsubsection{Cloud Operations}

The concept we are using for cloud architecture is AWS. AWS will work on various platforms of implementations at a time and its IAAS will support all the networking concepts and it will provide the high bandwidth and low fault tolerance architecture of the network. We can scale up and scale down.

\subsubsection{Natural Language Processing}

The concept of machine learning will be satisfied with using NLP to perform some prediction operations and whenever any video was recorded we can transfer that from video to text so that we can understand what's happening there. And we can forward that text information to the respective department.

\subsubsection{Results}

The results we got met our expectations and these are as follows. In this graph we have number of cases registered and what is the frequency of getting the same kind of issues as shown in Figure 8.

Figure 9 chart explains the monthly report we gathered.

\section{Conclusion}

The concept of generic architecture will create a mess in some of the technical things. And here we are proposing a generic architecture by taking risk but we try to achieve some things in this architecture with four-tier architecture and we are implementing both machine learning and internet of things with help of 


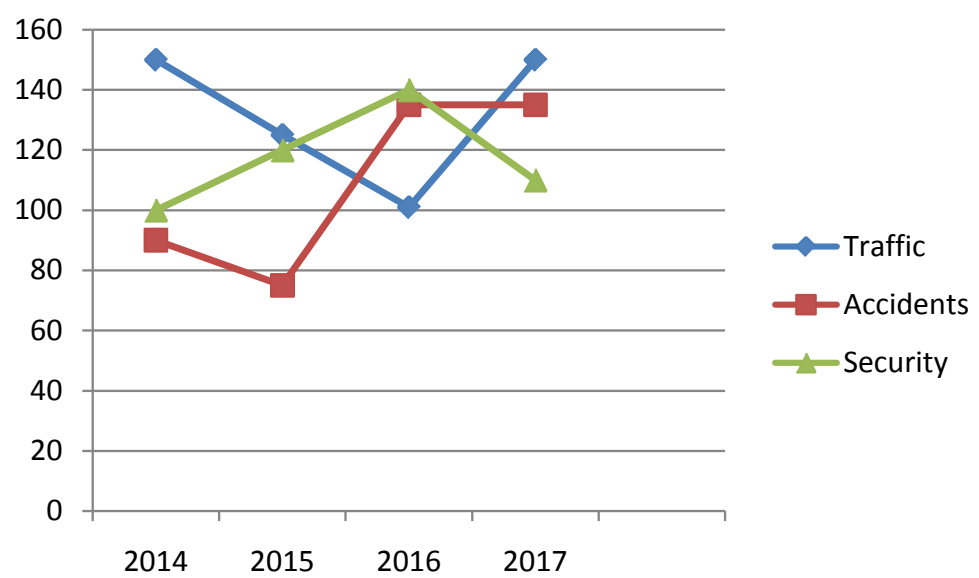

Figure 8. Results obtained by number of cases recorded from wearable device. Yearly Report.

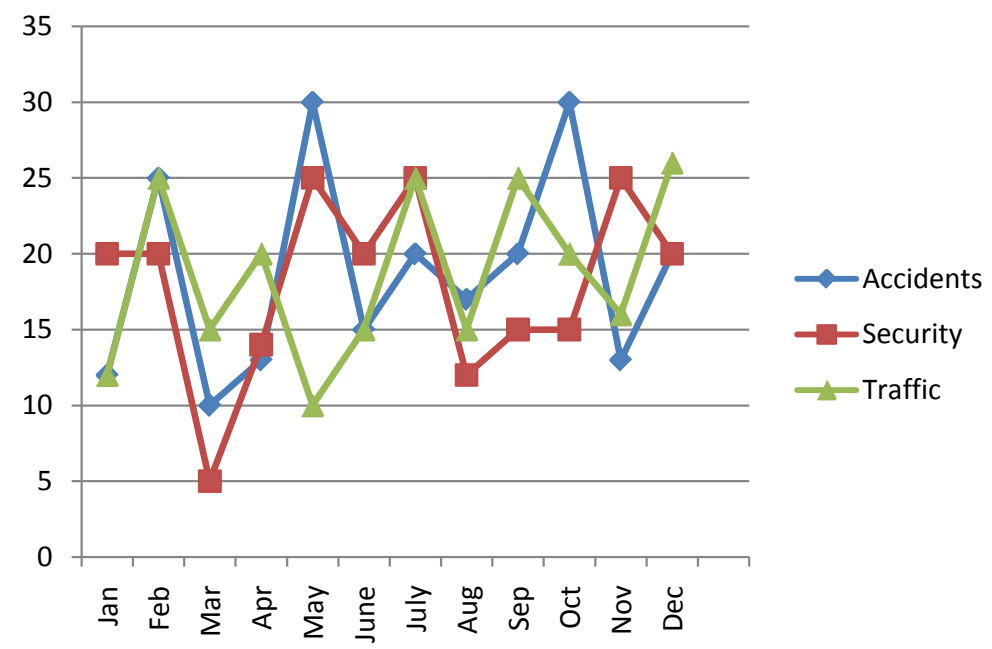

Figure 9. Results obtained by number of cases recorded from wearable device. Monthly Report.

cloud computing for data storage and access. Our architecture will help to protect the data and also will try to share environmental issues and urban area issues effectively. There is no privacy issue in this architecture because if anyone takes any wrong images, they are not even stored in the physical devices and they will be caught if they do any mischief things.

\section{Conflicts of Interest}

The authors declare no conflicts of interest regarding the publication of this paper.

\section{References}

[1] Ratti, C., Frenchman, D., Pulselli, R.M. and Williams, S. (2006) Mobile Landscapes: Using Location Data from Cell Phones for Urban Analysis. Environment and Planning B: Urban Analytics and City Science, 33, 727-748. 
https://doi.org/10.1068/b32047

[2] Kiukkonen, N., Blom, J., Dousse, O., Gatica-Perez, D. and Laurila, J. (2010) Towards Rich Mobile Phone Datasets: Lausanne Data Collection Campaign. Proc. ICPS, 1-7.

https://www.idiap.ch/ gatica/publications/KiukkonenBlomDousseGaticaLaurila-ic ps10.pdf

[3] Shilton, K. (2009) Four Billion Little Brothers?: Privacy, Mobile Phones, and Ubiquitous Data Collection. Communications of the ACM, 52, 48-53. https://doi.org/10.1145/1592761.1592778

[4] Fulk, G.D., Combs, S.A., Danks, K.A., Nirider, C.D., Raja, B. and Reisman, D.S. (2014) Accuracy of 2 Activity Monitors in Detecting Steps in People with Stroke and Traumatic Brain Injury. Physical Therapy, 94, 222-229.

https://doi.org/10.2522/ptj.20120525

[5] Han, B. and Srinivasan, A.E. (2012) Discovery: Energy Efficient Device Discovery for Mobile Opportunistic Communications. 2012 20th IEEE International Conference on Network Protocols (ICNP), Austin, TX, 30 October-2 November 2012, 1-10. https://doi.org/10.1109/ICNP.2012.6459980

[6] Lin, K., Kansal, A., Lymberopoulos, D. and Zhao, F. (2010) Energy-Accuracy Trade-Off for Continuous Mobile Device Location. Proceedings of the 8 th International Conference on Mobile Systems, Applications, and Services, San Francisco, California, 5-18 June 2010, 285-298. https://doi.org/10.1145/1814433.1814462

[7] Wilhelm, E., Zhou, Y., Zhang, N., Kee, J., Loh, G. and Tippenhauer, N. (2016) Sensg: Large-Scale Deployment of Wearable Sensors for Trip and Transportmode Logging. Proceedings of Transportation Research Board 95th Annual Meeting, Washington DC, 10-14 January 2016, 17 P.

[8] (2016) (SUTD MEC G) IT Repository. https://github.com/SUTDMEC

[9] Land Transport Authority (2015) Green Transportation. https://www.lta.gov.sg/content/dam/ltaweb/corp/PublicationsResearch/files/Report Newsletter/LTMP2013Report.pdf

[10] Monnot, B., et al. (2016) Inferring Activities and Optimal Trips: Lessons from Singapore's National Science Experiment. In: Cardin, M.A., Fong, S., Krob, D., Lui, P. and Tan, Y., Eds., Complex Systems Design, Management Asia, Vol. 426, Springer, Switzerland, 247-264. https://doi.org/10.1007/978-3-319-29643-2_19

[11] Mukhopadhyay, S.C. (2015) Wearable Sensors for Human Activity Monitoring: A Review. IEEE Sensors Journal, 15, 1321-1330. https://doi.org/10.1109/JSEN.2014.2370945

[12] Kelly, S.D.T., Suryadevara, N.K. and Mukhopadhyay, S.C. (2013) Towards the Implementation of IoT for Environmental Condition Monitoring in Homes. IEEE Sensors Journal, 13, 3846-3853. https://doi.org/10.1109/JSEN.2013.2263379

[13] Magno, M., Polonelli, T., Benini, L. and Popovici, E. (2015) A Low Cost, Highly Scalable Wireless Sensor Network Solution to Achieve Smart LED Light Control for Green Buildings. IEEE Sensors Journal, 15, 2963-2973. https://doi.org/10.1109/JSEN.2014.2383996

[14] Spachos, P. and Hatzinakos, D. (2016) Real-Time Indoor Carbon Dioxide Monitoring through Cognitive Wireless Sensor Networks. IEEE Sensors Journal, 16, 506-514. https://doi.org/10.1109/JSEN.2015.2479647

[15] Bi, Y., Lv, M., Song, C., Xu, W., Guan, N. and Yi, W. (2016) Autodietary: A Wearable Acoustic Sensor System for Food Intake Recognition in Daily life. IEEE Sensors 
Journal, 16, 806-816. https://doi.org/10.1109/JSEN.2015.2469095

[16] Li, G., Lee, B.-L. and Chung, W.-Y. (2015) Smartwatch-Based Wearable EEG System for Driver Drowsiness Detection. IEEE Sensors Journal, 15, 7169-7180. https://doi.org/10.1109/JSEN.2015.2473679

[17] Yang, H., Qin, Y., Feng, G. and Ci, H. (2013) Online Monitoring of Geological $\mathrm{CO}_{2}$ Storage and Leakage Based on Wireless Sensor Networks. IEEE Sensors Journal, 13, 556-562. https://doi.org/10.1109/JSEN.2012.2223210

[18] Kumar, A. and Hancke, G.P. (2015) A ZigBee-Based Animal Health Monitoring System. IEEE Sensors Journal, 15, 610-617. https://doi.org/10.1109/JSEN.2014.2349073

[19] Golding, A.R. and Lesh, N. (1999) Indoor Navigation Using a Diverse Set of Cheap, Wearable Sensors. 3rd International Symposium on Wearable Computers, San Francisco, 18-19 October 1999, 29-36. https://doi.org/10.1109/ISWC.1999.806640

[20] Lee, S.-W. and Mase, K. (2002) Activity and Location Recognition Using Wearable Sensors. IEEE Pervasive Computing, 1, 24-32. https://doi.org/10.1109/MPRV.2002.1037719

[21] Pirttikangas, S., Fujinami, K. and Nakajima, T. (2006) Feature Selection and Activity Recognition from Wearable Sensors. In: Ubiquitous Computing System, Springer, Berlin, 516-527.

[22] Ermers, M., Pärkkä, J., Mäntyjärvi, J. and Korhonen, I. (2008) Detection of Daily Activities and Sports with Wearable Sensors in Controlled and Uncontrolled Conditions. The IEEE Transactions on Information Technology in Biomedicine, 12, 20-26. https://doi.org/10.1109/TITB.2007.899496

[23] Subramanya, A., Raj, A., Bilmes, J.A. and Fox, D. (2012) Recognizing Activities and Spatial Context Using Wearable Sensors. https://arxiv.org/abs/1206.6869

[24] Huynh, D.T.G. (2008) Human Activity Recognition with Wearable Sensors. PhD Dissertation, Dept. Informatik, Technische Univ. Darmstadt, Darmstadt.

[25] Buechley, L., Eisenberg, M., Catchen, J. and Crockett, A. (2008) The LilyPad Arduino: Using Computational Textiles to Investigate Engagement, Aesthetics, and Diversity in Computer Science Education. Proceedings of the 2008 Conference on Human Factors in Computing Systems, Florence, 5-10 April 2008, 423-432.

[26] Sankaran, K., Zhu, M., Guo, X.F., Ananda, A.L., Chan, M.C. and Peh, L.-S. (2014) Using Mobile Phone Barometer for Low-Power Transportation Context Detection. Proceedings of the 12th ACM Conference on Embedded Network Sensor Systems, Memphis, 3-6 November 2014, 191-205.

[27] Zhao, F., et al. (2015) Exploratory Analysis of a Smartphone-Based Travel Survey in Singapore. Transportation Research Record: Journal of the Transportation Research Board, 2494, 45-56. https://doi.org/10.3141/2494-06

[28] Werner-Allen, G., Swieskowski, P. and Welsh, M. (2005) MoteLab: A Wireless Sensor Network Testbed. 4th International Symposium on Information Processing in Sensor Networks, Boise, 15 April 2005, 483-488.

[29] Taslidere, E., Cohen, F.S. and Reisman, F.K. (2011) Wireless Sensor Networks-A Hands-On Modular Experiments Platform for Enhanced Pedagogical Learning. IEEE Transactions on Education, 54, 24-33. https://doi.org/10.1109/TE.2010.2041235

[30] Kortuem, G., Bandara, A.K., Smith, N., Richards, M. and Petre, M. (2013) Educating the Internet-of-Things Generation. Computer, 46, 53-61.

https://doi.org/10.1109/MC.2012.390 
[31] Ball, T., Protzenko, J., Bishop, J., Moskal, M., de Halleux, J. and Braun, M. (2016) The BBC Micro: Bit Coded by Microsoft Touch Develop.

[32] Spanbauer, A., Wahab, A., Hemond, B., Hunter, I. and Jones, L. (2013) Measurement, Instrumentation, Control and Analysis (MICA): A Modular System of Wireless Sensors. IEEE International Conference on Body Sensor Networks, Cambridge, 6-9 May 2013, 1-5. 